\title{
Maximizing strain in miniaturized dielectric elastomer actuators
}

Samuel Rosset ; Oluwaseun Araromi and Herbert Shea

" Maximizing strain in miniaturized dielectric elastomer actuators ", Proc. SPIE 9430, Electroactive Polymer Actuators and Devices (EAPAD) 2015, 94301J (April 1, 2015); doi:10.1117/12.2083821; http://dx.doi.org/10.1117/12.2083821

Copyright 2015 Society of Photo-Optical Instrumentation Engineers. One print or electronic copy may be made for personal use only. Systematic electronic or print reproduction and distribution, duplication of any material in this paper for a fee or for commercial purposes, or modification of the content of the paper are prohibited. 


\title{
Maximizing strain in miniaturized dielectric elastomer actuators
}

\author{
Samuel Rosset, Oluwaseun Araromi, Herbert Shea \\ Ecole polytechnique fédérale de Lausanne, Switzerland
}

\begin{abstract}
We present a theoretical model to optimise the unidirectional motion of a rigid object bonded to a miniaturized dielectric elastomer actuator (DEA), a configuration found for example in AMI's haptic feedback devices, or in our tuneable RF phase shifter. Recent work has shown that unidirectional motion is maximized when the membrane is both anistropically prestretched and subjected to a dead load in the direction of actuation. However, the use of dead weights for miniaturized devices is clearly highly impractical. Consequently smaller devices use the membrane itself to generate the opposing force. Since the membrane covers the entire frame, one has the same prestretch condition in the active (actuated) and passive zones. Because the passive zone contracts when the active zone expands, it does not provide a constant restoring force, reducing the maximum achievable actuation strain. We have determined the optimal ratio between the size of the electrode (active zone) and the passive zone, as well as the optimal prestretch in both in-plane directions, in order to maximize the absolute displacement of the rigid object placed at the active/passive border. Our model and experiments show that the ideal active ratio is $50 \%$, with a displacement twice smaller than what can be obtained with a dead load. We expand our fabrication process to also show how DEAs can be laser-post-processed to remove carefully chosen regions of the passive elastomer membrane, thereby increasing the actuation strain of the device.
\end{abstract}

Keywords: Dielectric elastomer actuators, in-plane motion, optimization, hyperelasticity

\section{INTRODUCTION}

Among the different possibilities that allows using the electromechanical deformation of dielectric elastomer actuators (DEAs) to produce mechanical work, one particularly interesting configuration consists in using the in-plane expansion of the electrode to translate a rigid object attached to the dielectric elastomer. The resulting displacement occurs in the plane of the dielectric membrane. This configuration is used in the haptic feedback devices from Artificial Muscles Inc., in which a mass is put in motion by DEAs, in order to provide realistic vibrations. ${ }^{1}$ In our laboratory, we have been developing devices for mm-wave RF reconfigurable applications, in which a PCB with conducting zones is moved in-plane by a DEA. ${ }^{2}$ As a last example, the write cycle of O'Brien's Turing-complete DEA-based computer is achieved by moving a rigid body fixed to the actuator's membrane. ${ }^{3}$ For all these applications it is highly desirable to optimize the displacement of the rigid object placed on the membrane relative to the size of the device, in order to enhance the compactness of DEA systems. In particular, what is the optimum prestretch of the elastomeric membrane, and the best layout of the device?

Recently, progress has been made on the modelling of DEAs, using a strain energy density function to describe the hyperelastic behaviour of the elastomeric membrane. ${ }^{4}$ This has allowed to demonstrate and explain important behaviours, such as the suppression of the electromechanical instability (EMI), leading to gigantic actuation strain, ${ }^{5}$ or the possibility to obtain highly directional strains. ${ }^{6}$ Several studies have shown the importance of prestretch for the suppression of EMI and the achievement of large actuation strains, and have shown that a dead weight (i.e. constant force) is the optimal way of mechanically prestretching the dielectric membrane. ${ }^{7-9}$ In particular, Lu et al. have studied the impact of different prestretch conditions on vertically mounted stripeshaped DEAs under the influence of a weight pulling on the stripe vertically. ${ }^{9}$ The mass of the dead weight was used to set the value of the mechanical prestretch in the vertical direction, and the relation between the prestretch amplitude and maximal stretch was analysed for different prestretch configurations (equi-biaxial, uniaxial and anisotropic). The conclusion of the study was that the optimal prestretching configuration was a high prestretch in the transverse direction (which was held with stiff reinforcing fibres), and a low prestretch in the actuation direction (i.e. produced by a low dead weight).

contact author: samuel.rosset@a3.epfl.ch 


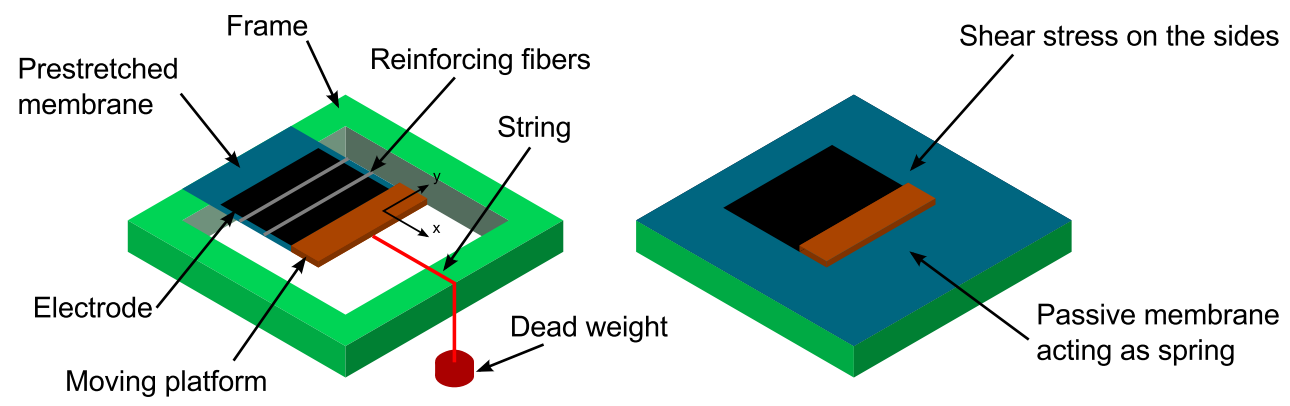

Figure 1. Left: Application of the concept of $\mathrm{Lu}$ et al. to the displacement of a moving platform, which would be fixed at one extremity of the membrane, and attached to the dead weight. Right: more realistic embodiment of the principle: the role of the dead weight is taken by a passive zone of the membrane acting as a spring. The membrane covers the complete frame, and the passive parts on the side of the platform have an influence on the actuation strain.

In this contribution, we apply the model of Lu et al. to the optimization of the displacement of a rigid movable platform fixed on a DEA. As the use of dead weights is impractical for this configuration, we use the elastomeric membrane to provide the counterbalancing force and look at the impact of having a non-constant force on the actuation amplitude. We also analyse what are the ideal prestretch conditions - both in the actuation direction and transverse direction - to maximize the displacement of the platform.

\section{GEOMETRY OF THE ACTUATOR}

The study of Lu et al. ${ }^{9}$ can be applied directly to a platform-shifting configuration, as the moveable rigid platform could be attached at the extremity of the DEA stripe, as depicted on figure 1 left. However, the figure also illustrates the limitation of this approach, especially for miniaturized devices. The use of a dead weight is highly impractical, because the device would work only in one orientation, and the attachment and force transmission system is hardy realisable on a small-scale device. Manipulating (prestretching and fixing) membranes of a few square $\mathrm{mm}$ is also very difficult: for small devices, prestretching is generally done by mechanically deforming a membrane of larger dimensions, and then gluing frames on the prestretched membrane. Consequently the membrane covers the complete surface of the frame, and a more realistic approach of a DEA that can move a rigid platform is shown on figure 1 right. The dead weight is replaced by the passive region of the membrane, which acts as a spring (a displacement-dependent force), and not as a constant force on the moveable platform. Additionally, the lateral sides of the platform and active zone are not free to move, but are but are constrained via the part of the passive region bonded to the frame. When the electrode is activated and the platform moves along the $\mathrm{x}$ direction, shear stress is generated in these zones, which will reduce the displacement compared to the unconstrained situation. For example, it can be intuitively understood that reducing the width of this lateral passive zone to 0 will prevent any displacement. Minimizing the negative impact of the lateral zone on the actuation strain calls for a large passive zone on the side, thus increasing the size of the actuator in the direction perpendicular to the actuation direction.

\subsection{Laser post-processing}

One possibility to remove the impact of the lateral passive zone is to post-process the actuator once the electrodes are applied, in order to cut the sides of the membrane, which can for example be done with a laser cutter (figure 2). Because the dielectric membrane is prestretched, it cannot simply be put under a laser to be cut. Indeed as soon as the cutting process starts, the freestanding membrane would move in an unpredictable manner due to the release of internal stress, and make it impossible to obtain a precise cut shape. It is necessary to support the membrane on the backside, in order to prevent it from moving during the laser post-processing. We have developed a process flow allowing to cut prestretched suspended PDMS membranes precisely and without damage (figure 3). Starting from the finished actuator (silicone membrane prestretched on a frame, electrodes printed, 

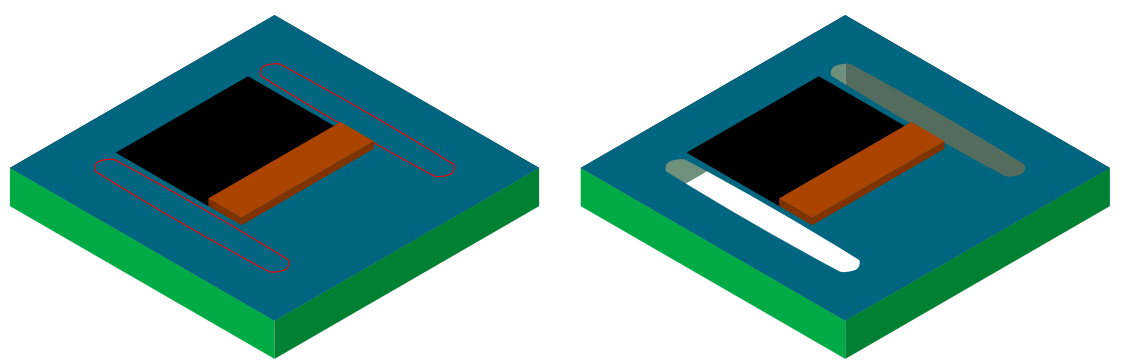

Figure 2. Post processing of the actuator can be made with a laser cutter to remove the passive membrane on the sides. Left: Cutting contours represented by red lines on the membrane. Right: resulting geometry after the cut.

a)

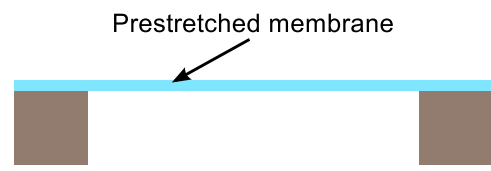

b)

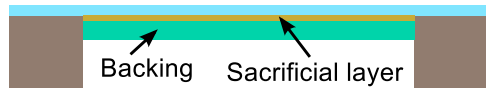

c)

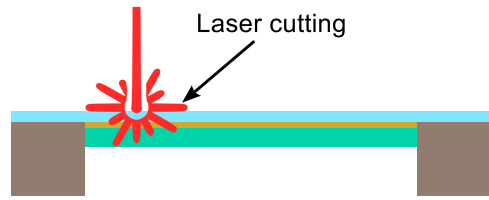

d)

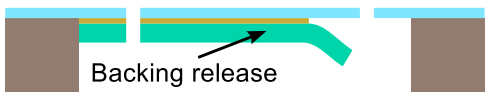

Figure 3. Process flow for the laser post-processing: starting from the finished device with prestretched membrane, electrodes and platform (a), a backing foil is applied using a water-soluble sacrificial layer as a glue (b). The membrane, held in place by the backing, is cut with a $\mathrm{CO}_{2}$ laser (c). Finally, the backing foil is removed by dissolution of the sacrificial layer in water $(\mathrm{d})$.

moveable platform fixed on the membrane, as well as the lateral stiffeners, if required), a flexible foil (0.1 mm PET foil) is fixed to the membrane backside using a water-soluble sacrificial material (PVA, polyvinylalcohol) as an adhesive between the membrane and the foil. Once the sacrificial layer has dried, the membrane is cut using a $60 \mathrm{~W} \mathrm{CO} \mathrm{CO}_{2}$ laser (Speedy 300, Trotec), with $5.5 \%$ of the laser power at $1000 \mathrm{~Hz}$, and with a cutting speed of $35 \mathrm{~mm} / \mathrm{s}$. The precise alignment between the laser cuts and the actuator is made using a registration camera and the software i-cut vision pro. Because the silicone membrane is fixed to the unstretchable PET backing through the PVA soluble layer, it cannot relax and move while being cut, which ensures a very precise geometry. The cuts are designed with large radii of curvature at the edges, to avoid tearing the membrane once it is allowed to relax. To remove the backing, the actuator is immersed into warm water, in order to dissolve the PVA layer.

The application of this process flow is illustrated in figure 4 for the case of an actuator designed to move a $35 \times 35 \mathrm{~mm}^{2}$ platform. The PET backing is fixed to the finished actuator (prestretched membrane, compliant electrodes, movable platform (green shaded area) and two stiffenenrs) using a 5\% PVA solution in water. Once the PVA layer has dried, the passive part of the membranes located on the side of the platform and electrodes is cut by laser. The registration camera and associated software allows to precisely align the cut with the existing actuator, as shown in figure $4 \mathrm{~B}$ ), where the cut is avoiding the anchor points of the stiffener. Finally the PET backing is removed by dissolution of the PVA layer in warm water. This allows the membrane to relax. The stiffeners help preventing the relaxation of the membrane, as can be seen by the difference of the membrane side profile with and without stiffener in the electrode region (in the platform region, there is no relaxation possible, because the platform is fixed to the membrane, which prevents relaxation).

The laser post-processing of the actuator allows removing the passive membrane on the sides of the the moving platform and the electrodes, thus mimicking the situation modelled by $\mathrm{Lu}$ et al. ${ }^{9}$ One of the main differences, however, is the constant force provided by the dead weight, which is replaced in our case by the passive region of the membrane and becomes a strain-dependent force. In the next section, the impact of this strain-dependent force on the stretch of the electrodes and displacement of the platform is investigated. 

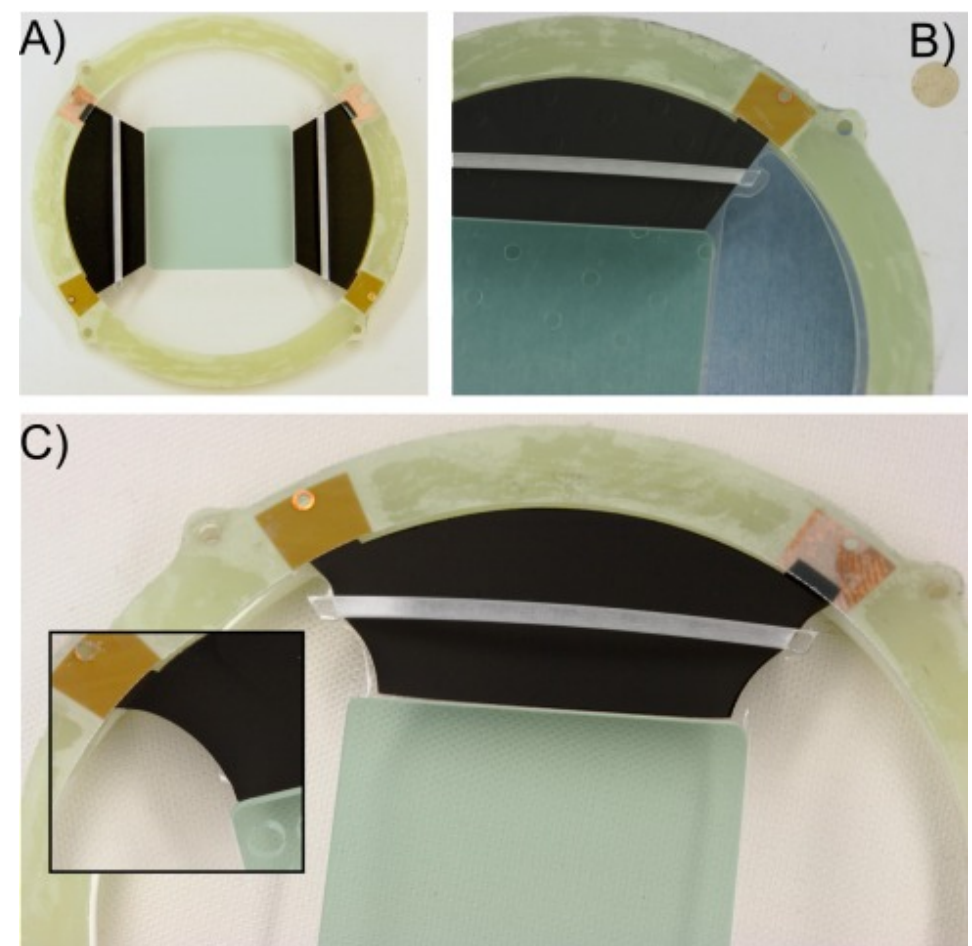

Figure 4. Illustration of the laser post-processing: A) Finished actuator with prestretched membrane, electrodes, moving platform (green shaded area) and one stiffener per electrode. B) After cutting with the laser. The cut can be precisely aligned to conform to the geometry of the design. The PET backing has holes, in order to provide access to the sacrificial layer for the water. C) After release of the PET backing in water. Inset: electrode side profile in the absence of the stiffener.

\section{DISPLACEMENT OPTIMIZATION}

We consider an actuator formed by 3 separate regions (figure 5) an electrode of length $l_{a}$, a passive zone of length $l_{p}$, and a rigid platform separating these two zones. The platform is considered to be weightless and infinitely stiff. Consequently the platform doesn't deform, but is just displaced by the electrode. Its length has no influence on the behaviour of the device. To simplify, we consider a zero width platform, so that the total length of the membrane is the sum of the electrode and passive zone: $l_{t o t}=l_{a}+l_{p}$. Likewise, we also consider infinitely thin stiffeners. Stiffeners are required to keep the aspect ratio of the free standing membrane smaller than 1:5 (direction 1:direction 2) so that the prestretch in direction 2 doesn't relax. For a real-world device, the length of the platform and the length of all of the stiffeners must be added to the active and passive zones to get the total length of the device. The membrane is prestretched along direction 1 and 2 with prestretch ratios $\lambda_{1 p s}$ and $\lambda_{2 p s}$. We assume that the prestretch state in the membrane is not affected by the laser cutting (i.e. stiffeners are added on the active and passive zones if needed).

Given an elastomer with a known stress-stretch behaviour described by a strain energy density function $w$, the main degrees of freedom which will influence the displacement of the platform are the prestretches in direction 1 and 2 , and the length of the electrode with respect to the total length, which we call the active ratio: $r_{a}=l_{a} /\left(l_{a}+l_{p}\right)$. We look at different actuator configurations (different values of $r_{a}, \lambda_{1 p s}$ and $\lambda_{2 p s}$ ) and calculate the maximal achievable stretch of the electrode. The maximal achievable stretch is defined as the stretch observed before a failure occurs. Three main failures modes are taken into account: dielectric breakdown, electromechanical instability and loss of tension in direction 1 (which would cause the platform to start moving out of plane). The stretch is obtained by solving the equations of state of DEAs, ${ }^{4}$ taking into account the elastic energy stored into the active and passive zones of the membrane, and the electrostatic energy stored into the active zone when a voltage is applied. 


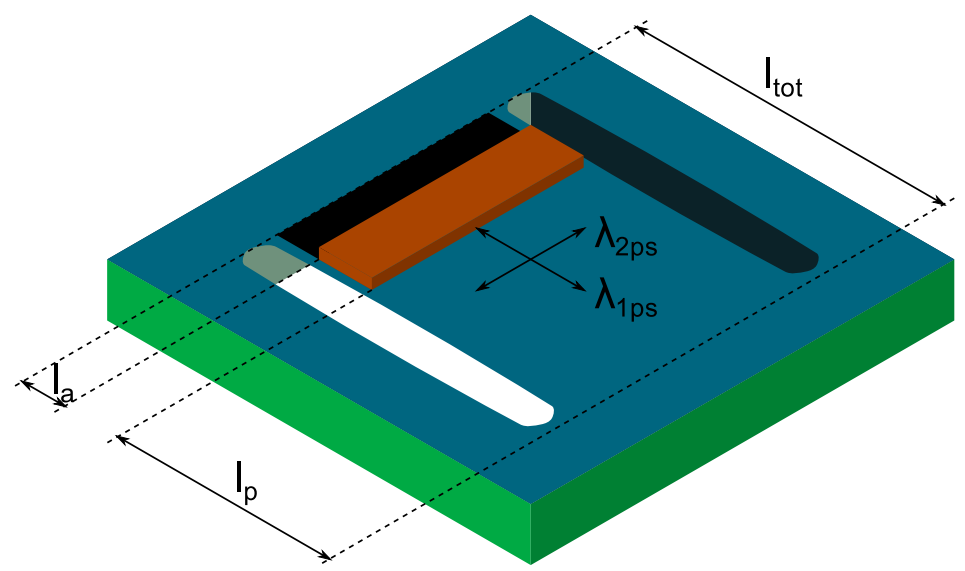

Figure 5. Model of the actuator: A suspended membrane is separated in 3 regions: an electrode of length $l_{a}$, a passive zone of length $l_{p}$, and a rigid platform separating the two regions. The membrane is prestretched along the direction 1 and 2 with prestretch ratios $\lambda_{1 p s}$ and $\lambda_{2 p s}$. The displacement of the platform occurs along direction 1 . One of the important design parameters is the active ratio $r_{a}$, i.e. the length of the electrode with respect to the total length of free membrane: $r_{a}=l_{a} /\left(l_{a}+l_{p}\right)$.
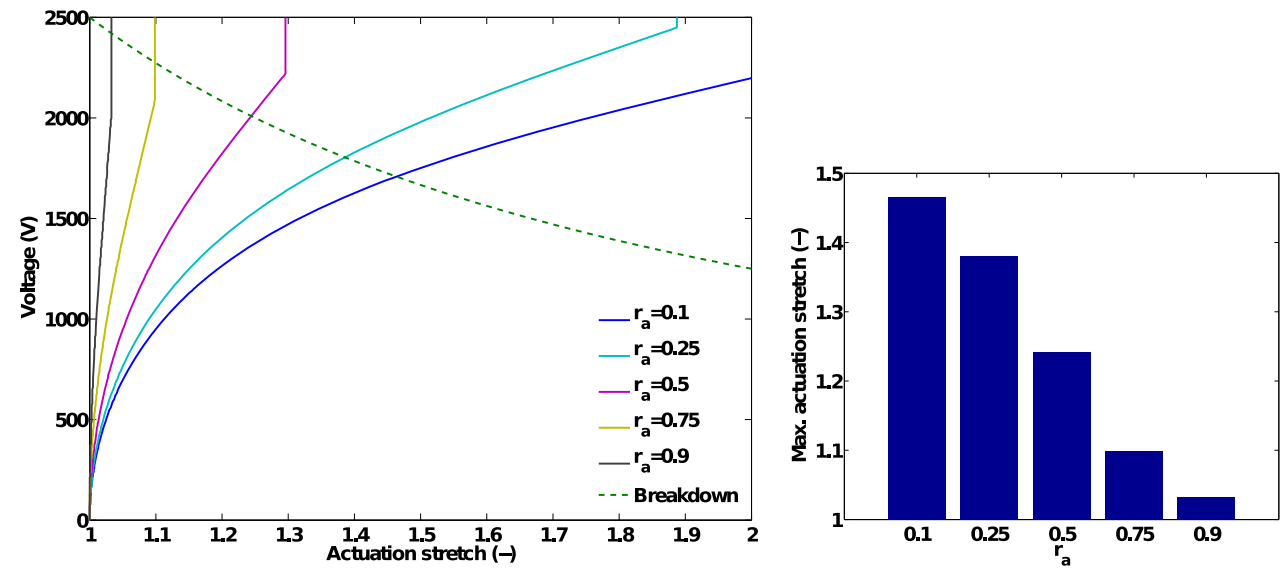

Figure 6. Left: Voltage versus actuation stretch curves for different values of active ratio $r_{a}$. The green dashed line represents the dielectric breakdown limit. The vertical portion of the curve represents the loss of tension in direction 1. Right: maximal electrode stretch for the different active ratios.

For the numerical application, we consider the silicone elastomer Sylgard 186 from Dow Corning, which we model using the Gent hyper-elastic model. ${ }^{10}$ The value for the 2 model parameters were obtained by fitting the model on experimental traction test data. Because of the actuator layout, where the stretch along the direction 2 is constant, the traction test was conducted in pure shear mode, using a sample whose initial length was 10 times smaller than its width. The fitting of the Gent model on the experimental data leads to $\mu=0.16 \mathrm{MPa}$ and $J=18.3 .{ }^{11}$ In addition, we define the maximal electric field that the elastomer can sustain to $100 \mathrm{~V} / \mu \mathrm{m}$. We consider membranes with a thickness of $25 \mu \mathrm{m}$ after prestretch, and a relative permittivity $\epsilon_{r}=2.5$ for the silicone.

\subsection{Optimization of the active ratio}

In a first step, we look at the impact of the active ratio $r_{a}$. We fix the prestretch value to $\lambda_{1 p s}=1$ and $\lambda_{2 p s}=2$, and calculate the stretch of the active zone for different values of active ratio $r_{a}$ (figure 6 ). The maximal stretch is defined as the stretch reached at the dielectric breakdown field or when loss of tension occurs in the actuation 

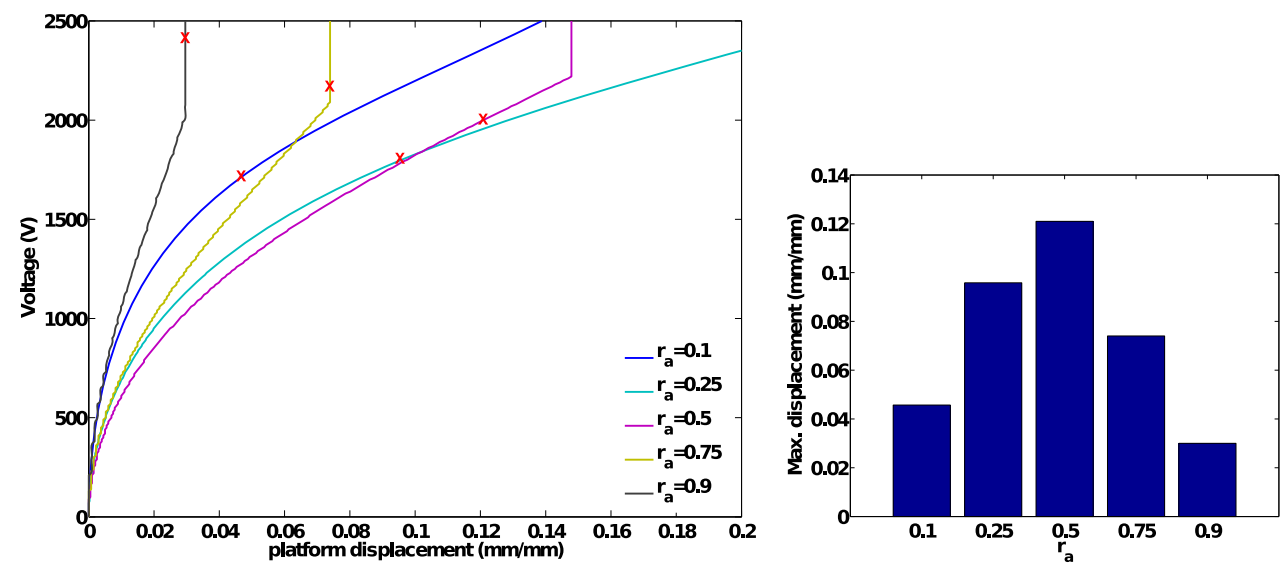

Figure 7. Left: Voltage versus normalized platform displacement curves for different values of active ratio $r_{a}$. The red crosses represent the dielectric breakdown limit for each of the curves. Right: maximal platform displacement for the different active ratios.

direction. The dielectric breakdown field is indicated by the green dashed curve on the graph, and the loss of tension is indicated by a vertical line on the curves. The largest stretch is obtained for the lowest active ratio. This corresponds to a very large passive zone length compared to the size of the electrode. This is the constant force case, which is considered to be the optimal pretretch situation. ${ }^{7-9}$ With increasing values of the active ratio $r_{a}$, the maximal stretch decreases, due to the diminishing force provided by the passive zone when the platform moves. If the ratio becomes too large, loss of tension in the actuation direction occurs before dielectric breakdown. Even though the maximal stretch is maximized for very small values of $r_{a}$, this solution is not optimal because it means that 1) either the total device becomes very large to account for the long passive zone, or 2) if the size of the device is fixed, then the size of the electrode becomes small, and leads to a small displacement of the platform.

Consequently, a better metric to analyse the impact of the active ratio $r_{a}$ is the displacement of the platform, rather than the stretch of the active zone. The displacement of the platform $d_{p}$ is given by:

$$
d_{p}=l_{\text {tot }} r_{a}\left(\lambda_{a}-1\right)
$$

where $\lambda_{a}$ is the actuation stretch of the active zone, as shown on figure 6 . We choose $l_{\text {tot }}$ to be $1 \mathrm{~mm}$ in order to obtain a normalized displacement of the platform per mm of total device length.

The normalized displacements of the moving platform (figure 7) are quite different from the stretch of the electrode shown previously (figure 6), because the initial length of the electrode influences the results, and a small $r_{a}$, which leads to a large stretch also corresponds to a small electrode size. It can be seen that there is an optimum, and that a small value of $r_{a}$ is not recommended. The exact optimum value of $r_{a}$ depends on the hyperelastic model used and the value of the parameters. For the present case, it is $44 \%$, however, the displacement difference between $44 \%$ and $50 \%$ active ratio is quite small, and the latter situation allows to make push-pull actuators, with active zones on both sides of the platform, such as on the actuator shown on figure 4 . This allows to double the displacement and to have a symmetric behaviour between the two actuators.

The maximal actuation stretch of the electrode in the case of a constant force acting on the platform (large passive zone) is 1.46 , whereas for the configuration with a $50 \%$ active ratio, the maximal stretch drops to 1.24 . The actuation strain is consequently divided by a factor 2 between a case using a constant force pulling on the platform (dead weight or very large passuve zone) and a case with a $50 \%$ active ratio. However, the latter configuration leads to a larger displacement relative to the total size of the device, and the possibility to have bidirectional actuation allows to compensate for this loss. 

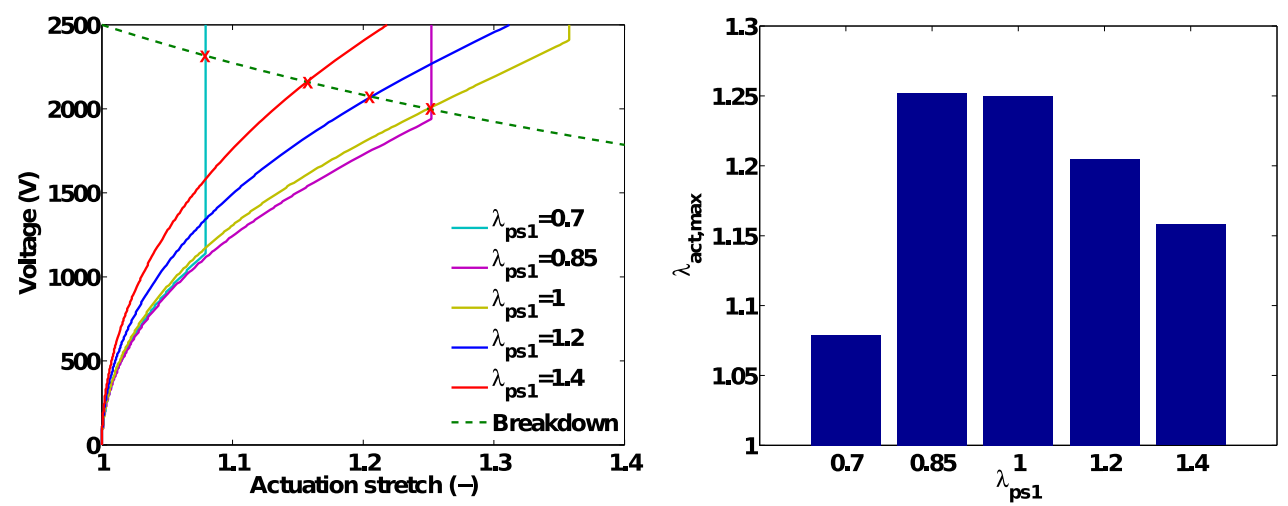

Figure 8. Left: Actuation stretch for an active ratio of $50 \%$ and a prestretch in the transverse direction of $\lambda_{2 p s}=2.46$, and different values of prestetch in the actuation direction. The red crosses indicate the dielectric breakdown, and therefore the maximal actuation stretch. Right: maximal actuation stretch before failure for the different values of $\lambda_{1 p s}$

\subsection{Optimization of the prestretch}

With an optimal active ratio fixed at 50\%, the next step consists in optimizing the prestretch of the membrane both in the actuation direction and in the transverse direction. The exact values of the optimal prestretch depend on the hyperelastic model and parameters used to model the dielectric membrane. It is however interesting to have a look on the influence of the prestretch in the actuation direction $\left(\lambda_{1 p s}\right)$ over the behaviour of the actuator. Using the same parameters for the Gent model used previously, the optimal prestretch in the transverse direction is $\lambda_{2 p s}=2.46$.

We plot the Voltage-actuation stretch curve for this partiular value of $\lambda_{2 p s}$, an active ratio of $50 \%$, and different values of prestretch in the actuation direction (figure 8). It can be seen that for a low $\lambda_{1 p s}$ value, the maximal stretch is limited by loss of tension in the actuation direction, as evident for the case with $\lambda_{1 p s}=0.7$ : loss of tension occurs well before dielectric breakdown. The highest maximal actuation stretch is obtained for the situation in which the dielectric breakdown voltage coincides with the loss of tension in direction 1 . However this situation is undesirable, as out-of-plane displacement of the platform is probable at high driving voltage, due to the small amount of residual mechanical tension remaining in the membrane in this case (although we consider a weightless platform here for simplification, in real-world applications, the platform has a weight, and it is therefore desirable to keep a certain amount of mechanical stress in the membrane to avoid out-of plane deflection). It can be seen that for a slightly higher prestretch $\left(\lambda_{1 p s}=1\right)$, the maximal stretch is only slightly lower, but the voltage at which loss of tension occurs far exceeds the dielectric breakdown voltage, thus ensuring that the membranes always remains under mechanical tension. In addition, having $\lambda_{1 p s}=1$ also presents a practical advantage, as it makes prestretching easier, one of the dimensions of the membrane being kept constant during the process. For larger values of $\lambda_{1 p s}$, the maximal stretch decreases.

\section{CONCLUSIONS}

DEAs that can move a rigid platform in-plane cannot rely on a dead weight to provide a constant force pulling on the platform if the device must be of relatively small scale and/or functional in any orientation. Instead, it is the prestretched dielectric membrane itself, which acts as a spring pulling on the platform, with the consequence that the force it provides is not constant, but dependent on the displacement of the platform. To suppress the influence of the membrane located on the sides of the platform, electrodes and passive spring, the device can be post-processed by laser to cut the membrane on the sides.

The fact that the passive zone of the membrane provides a force whose amplitude depends on the platform position (as opposed to a constant force provided by a dead weight) leads to a reduction of the maximum actuation stretch that can be obtained from a device in the optimal configuration. Because the optimal geometrical configuration is obtained for a situation where the electrode and passive regions have the same dimensions (active ratio $r_{a}=0.5$ ), electrodes can also be applied on the passive zone to obtain a push-pull configuration, the platform being moved in one direction or the other depending on which actuator is activated, thus compensating for the factor two loss induced by the use of a displacement-dependent counter-force. 
In addition to the active ratio $r_{a}$, the prestretch of the membrane must also be optimized. The exact optimal parameters depends on the hyperelastic stretch-stress behaviour of the dielectric, but it is particularly important to avoid loss of tension in the actuation direction, as this drastically reduces the maximal actuation stretch. The theoretical optimum is achieved for a $\lambda_{1 p s}$ such that the loss of tension in direction 1 occurs exactly at the maximal admissible electric field. However in practice it is better to have a slightly higher $\lambda_{1 p s}$, as this allows keeping the membrane under mechanical tension for the complete admissible voltage range. Consequently, for the silicone elastomer considered here (Sylgard 186 from Dow Corning), the optimal prestretch parameters are $\lambda_{1 p s}=1$ and $\lambda_{2 p s}=2.46$, and for an active ratio $r_{a}=0.5$, it should be possible to move the platform by $0.125 \mathrm{~mm}$ per total length of actuator (active plus passive zone), i.e. $0.25 \mathrm{~mm}$ per $\mathrm{mm}$ of electrode length with a driving electric field of $100 \mathrm{~V} / \mu \mathrm{m}$. If the prestretching conditions do not coincide with the optimal parameters, then the achievable actuation strain drops sharply, especially if loss of tension in the actuation direction occurs at a lower field than the maximal admissible field, which can happen if the membrane prestretch in the actuation direction is too low.

\section{ACKNOWLEDGMENTS}

This work has been partially funded by the Swiss National Science Foundation grant 200020-153122.

\section{REFERENCES}

1. S. J. Biggs and R. N. Hitchcock, "Artificial muscle actuators for haptic displays: system design to match the dynamics and tactile sensitivity of the human fingerpad," in Proceedings of SPIE - The International Society for Optical Engineering, Proc. SPIE 7642, pp. 76420I-76420I-12, 2010.

2. S. Araromi, P. Romano, S. Rosset, J. Perruisseau-Carrier, and H. Shea, "A tunable millimeter-wave phase shifter driven by dielectric elastomer actuators," in Proceedings of SPIE, 9056, p. 90562M, 2014.

3. B. M. O'Brien and I. A. Anderson, "An artificial muscle computer," Applied Physics Letters 102(10), p. 104102, 2013.

4. Z. Suo, "Theory of dielectric elastomers," Acta Mechanica Solida Sinica 23(6), pp. 549-578, 2010.

5. C. Keplinger, T. Li, R. Baumgartner, Z. Suo, and S. Bauer, "Harnessing snap-through instability in soft dielectrics to achieve giant voltage-triggered deformation," Soft Matter 8, pp. 285-288, 2012.

6. S. Akbari, S. Rosset, and H. R. Shea, "Improved electromechanical behavior in castable dielectric elastomer actuators," Applied Physics Letters 102(7), p. 071906, 2013.

7. X. Zhao and Z. Suo, "Method to analyze electromechanical stability of dielectric elastomers," Applied Physics Letters 91(6), p. 061921, 2007.

8. J. Huang, T. Li, C. Chiang Foo, J. Zhu, D. R. Clarke, and Z. Suo, "Giant, voltage-actuated deformation of a dielectric elastomer under dead load," Applied Physics Letters 100(4), p. 041911, 2012.

9. T. Lu, J. Huang, C. Jordi, G. Kovacs, R. Huang, D. Clarke, and Z. Suo, "Dielectric elastomer actuators under equal-biaxial forces, uniaxial forces, and uniaxial constraint of stiff fibers," Soft Matter 8(22), pp. 6167-6173, 2012.

10. A. N. Gent, "A new constitutive relation for rubber," Rubber Chemistry and Technology 69, pp. 59-61, March 1996.

11. S. Rosset, O. A. Araromi, J. Shintake, and H. R. Shea, "Model and design of dielectric elastomer minimum energy structures," Smart Materials and Structures 23(8), p. 085021, 2014. 